\title{
Sleep Quality and Psychological Adjustment in Chronic Fatigue Syndrome
}

\author{
Myrtis Fossey, ${ }^{1,3}$ Eva Libman, ${ }^{1,3,5,6}$ Sally Bailes, ${ }^{1}$ Marc Baltzan, ${ }^{2,5}$ \\ Ronald Schondorf, ${ }^{1,5}$ Rhonda Amsel, ${ }^{5}$ and Catherine S. Fichten ${ }^{1,4,5}$
}

Accepted for publication: January 7, 2004

Without specific etiology or effective treatment, chronic fatigue syndrome (CFS) remains a contentious diagnosis. Individuals with CFS complain of fatigue and poor sleep-symptoms that are often attributed to psychological disturbance. To assess the nature and prevalence of sleep disturbance in CFS and to investigate the widely presumed presence of psychological maladjustment we examined sleep quality, sleep disorders, physical health, daytime sleepiness, fatigue, and psychological adjustment in three samples: individuals with CFS; a healthy control group; and individuals with a definite medical diagnosis: narcolepsy. Outcome measures included physiological evaluation (polysomnography), medical diagnosis, structured interview, and self-report measures. Results indicate that the CFS sample had a very high incidence (58\%) of previously undiagnosed primary sleep disorder such as sleep apnea/hypopnea syndrome and restless legs/periodic limb movement disorder. They also had very high rates of self-reported insomnia and nonrestorative sleep. Narcolepsy and CFS participants were very similar on psychological adjustment: both these groups had more psychological maladjustment than did control group participants. Our data suggest that primary sleep disorders in individuals with CFS are underdiagnosed in primary care settings and that the psychological disturbances seen in CFS may well

1S.M.B.D.-Jewish General Hospital, Montreal, Quebec, Canada.

${ }^{2}$ Mount Sinai Hospital, Montreal, Quebec, Canada.

${ }^{3}$ Concordia University, Montreal, Quebec, Canada.

${ }^{4}$ Dawson College, Montreal, Quebec, Canada.

${ }^{5}$ McGill University, Montreal, Quebec, Canada.

${ }^{6}$ To whom correspondence should be addressed at ICFP - Department of Psychiatry, Jewish General Hospital, 4333 Cote Ste Catherine Road, Montreal, Quebec, Canada H3T 1E4; e-mail: eva.libman@mcgill.ca. 
be the result of living with a chronic illness that is poorly recognized or understood.

KEY WORDS: insomnia; nonrestorative sleep; chronic fatigue syndrome (CFS); narcolepsy; primary sleep disorder; sleep apnea/hypopnea syndrome; restless legs/periodic limb movement disorder; psychological adjustment; psychopathology; health.

Chronic fatigue syndrome (CFS) is a functional disorder characterized by debilitating daytime fatigue. CFS has a chronic course (Fukuda et al., 1994) with no specific etiology or pathophysiology (cf. Kirmayer and Robbins, 1991), no single diagnostic test (Komaroff and Fagioli, 1996), and no consistently effective treatment (Cleare, 2003). The current procedure for diagnosing CFS is one of elimination, and patients with CFS are often told that they are suffering from a psychological problem such as a somatoform disorder or depression (David et al., 1988; Plioplys, 2003). Patients commonly report that prior to their illness, they were unusually physically vigorous and productive (Komaroff and Fagioli, 1996). Although there continues to be some controversy over the existence of CFS as a valid diagnosis, what remains indisputable is that many individuals in their 30 s and 40 s are disabled and distressed by a condition which causes them to limit their activities, to be lost from the workforce (Schondorf and Freeman, 1999), and to be a burden on the healthcare system (Komaroff, 1990; Wessely, 1995). CFS support groups lobby for disability coverage and insurance companies fight the claims and neither the public nor the medical communities agree on CFS as a real clinical entity (Caplan, 1998).

It seems increasingly unlikely that CFS is caused by a single, as yet unidentified, disease process. Since the symptoms of CFS typically persist for years, the original etiological factors may have either resolved or become irrelevant. Possibly, the fatigue symptoms have become autonomous, resulting in fatigue perseveration in which factors other than the original etiological ones operate to maintain the fatigue cycle. This argues for symptom-based intervention rather than trying to find an effective treatment for CFS as a single disease entity. This latter approach, the traditional one, has been largely ineffective.

Rather than attempting to identify structural and biological characteristics common to all people diagnosed with CFS, we believe that a productive alternative is to develop criteria that differentiate major subgroups based on symptom clusters. This approach has the advantage of targeting the disabling symptoms specifically. For example, recent work using a symptom-based approach has identified individuals who have CFS accompanied by orthostatic intolerance. Although orthostatic intolerance has been identified in 40\% of individuals (Schondorf et al., 1999; Schondorf and Freeman, 1999), to date, we know of no systematic study of the impact of regulating orthostatic disorder on chronic fatigue symptoms. 
A sample of individuals with CFS was included as part of a larger investigation in our laboratory where the focus was on distinguishing sleepiness from fatigue. A serendipitous finding was the high rate of sleep disorder such as sleep apnea/hypopnea syndrome, restless legs syndrome/periodic limb movement disorder (RLS/PLMD), and insomnia in the CFS sample (Bailes et al., 2001). A subsequent study (Bailes et al., 2003) confirmed these results and corroborated findings of relatively high rates of sleep disruption in CFS both in classic (e.g., Moldofsky, 1986; Moldofsky and Scarisbrick, 1976) and more recent studies (e.g., Le Bon et al., 2000).

There is a growing body of research suggesting that CFS may be caused or maintained by desynchonization or dysregulation of neuroendocrine functions which drive the sleep/wake cycle (Steiger, 2002). It is notable that some of the major presenting complaints of persons with CFS (e.g., daytime fatigue, difficulty with memory and concentration, insomnia, and nonrestorative sleep) are related to the sleep disruption and problems with daytime functioning common to individuals with primary sleep disorder and insomnia (Bailes et al., 2003). In addition, there is some suggestion in the literature of a desynchronization of the temperature and melatonin circadian rhythms-important markers in the sleep-wake cycle (Williams et al., 1996).

Documenting the presence and nature of sleep disorders in the CFS population could contribute to a better understanding of CFS and, possibly, yield more effective treatment strategies. For example, there are well established and widely used effective medical treatments for sleep apnea/hypopnea syndrome and RLS/PLMD (e.g., Montplaisir and Godbout, 1989), and both primary (e.g., Bootzin and Nicassio, 1978; Morin et al., 2003) and secondary (Lichstein et al., 2001) insomnia have been shown to respond well to cognitive-behavioral therapy. The mechanisms of human circadian system pacemakers and their entrainment are relatively well understood (Honma et al., 2003) and suggest interventions to resynchronize disrupted biological rhythms.

With respect to the widely held belief that individuals with CFS are depressed, anxious, and generally psychologically maladjusted, another link with sleep quality may be made. It has been shown that sleep disruption and sleep deprivation activate the dominant physiological pattern associated with exposure to stressful circumstances, largely involving the hypothalamicpituitary-adrenal (HPA) stress axis and the sympathetic nervous system (Leese et al., 1996; Spath-Schwalbe et al., 1992). Conversely, administering the stress hormones normally secreted during the physiological activation process (e.g., corticotropic-releasing hormone $(\mathrm{CRH})$ and adrenocorticotro pic hormone (ACTH)) impair sleep quality (Van Reeth et al., 2000). In addition, Van Reeth et al. noted that excess stress arousal/activation adversely affects immune functions and disturbs sleep and waking rhythms. This raises the possibility that sleep disorder may be implicated in both 
daytime functioning aspects as well as in the physical and psychological health aspects of CFS.

In keeping with the symptom-based approach, in the present investigation the goals were (1) to assess the nature and prevalence of sleep disturbance in CFS and (2) to investigate the widely presumed presence of psychological maladjustment in CFS. To accomplish this we studied sleep quality, sleep disorders, physical health, daytime sleepiness and fatigue, and psychological adjustment in three samples: individuals with CFS, a healthy control group, and individuals with narcolepsy. Outcome measures include physiological evaluation (polysomnography (PSG)), medical diagnosis, structured interview, and self-report measures. Narcolepsy was selected for comparison because this is a disabling sleep disorder which has only relatively recently been clearly identified as a neurological disease rather than a psychiatric one (cf. Siegel, 2000). In individuals with narcolepsy there is a profound reduction in the number of neurons in the hypothalamus containing hypocretin; this has been shown to play an important role in the regulation of sleep (Taheri et al., 2002). Individuals with narcolepsy experience frequent and unwanted sleepiness during wakefulness and they tend to awaken more frequently during sleep as well. Moreover, there is recent evidence that hypocretin plays an important role in the regulation of ACTH and cortisol (Kok et al., 2002; Taylor and Samson, 2003). Since the identified neurological lesions also involve an integral part of the HPA axis, the disorder has a number of important elements in common with CFS.

Compared to the control group, we expected the CFS sample to have a significantly higher rate of diagnosed medically based sleep disorders, more psychological maladjustment, more problems with daytime functioning (sleepiness and fatigue) and poorer self-reported health functioning and insomnia symptoms. We made no hypotheses about the narcolepsy sample.

\section{METHOD}

\section{Participants}

Participants were 37 individuals with CFS ( 31 females, 6 males, mean age $=37, \mathrm{SD}=15), 24$ individuals with narcolepsy ( 15 females, 5 males, mean age $=44, \mathrm{SD}=9)$, and 24 individuals $(17$ females, 7 males, mean age $=$ $40, \mathrm{SD}=9$ ) with no diagnosed medical or psychiatric condition (control group).

The CFS sample was recruited from physician referrals and CFS support groups. For each participant, two independent assessments of CFS were made. Participants arrived with a diagnosis from their own physician. The 
research team physician confirmed the original CFS diagnosis by using a standardized diagnostic instrument based on Fukuda et al.'s (1994) diagnostic criteria. None had ever been referred to a sleep laboratory and none had been diagnosed with a primary sleep disorder such as sleep apnea/hypopnea. Individuals with narcolepsy were recruited from physician referrals. Control group participants were recruited from the community through posters, announcements, and personal contacts.

All potential participants were volunteers. They were screened for comorbid diagnoses and excluded if (1) they suffered from a current major psychiatric illness; (2) had another medical condition related to fatigue, sleepiness, arthralgia, or insomnia (other than fibromyalgia, which was not excluded); (3) they were taking medication that interferes with sleep or causes fatigue or sleepiness; and (4) they were working rotating/split shifts or recently traveled across time zones. To the extent possible, Control group participants were selected from the same age group as those in the two clinical samples. The mean years of education for the three samples ranged from 15 to 16 years of schooling; the standard deviation was 4 . There were no significant differences among groups on age, years of education, or gender ratio.

\section{Diagnosis of Medically Based Sleep Disorder}

Diagnosis was carried out by a certified respirologist following polysomn ographic (PSG) assessment in accordance with the International Classification of Sleep Disorders of the American Sleep Disorders Association (Diagnostic Classification Steering Committee, 1990).

Participants were monitored in a supervised sleep laboratory from 10:00 PM to 7:00 AM. Monitoring included three leads EEG, EOG, bilateral anterior tibialis and chin EMG, ECG, pulse oximetry, nasal and oral airflow with thermistor and nasal pressure cannulae, and respitrace bands for measurement of respiratory effort. Leg movements, apnea events, and associated arousals were scored manually according to the scoring rules established by the Atlas Task Force of the American Sleep Disorders Association (1993) and the International Classification of Sleep Disorders (Diagnostic Classification Steering Committee, 1990).

Apnea was defined as cessation of breathing lasting 10 or more seconds with a frequency of more than five times per hour. Hypopneas were scored when there was $40 \%$ or more decrease in airflow with $2 \%$ or more oxygen desaturation. Periodic Limb Movement Disorder (PLMD)/Restless Legs Syndrome (RLS) was scored in cases of repetitive episodes of muscle contraction (0.5- to 5-s duration) or when awakenings were associated with the movements. 


\section{Determination of Insomnia}

Insomnia was self-defined in response to the following Yes/No question, "Do you have insomnia?" The type of insomnia (cf. American Psychiatric Association, 1994) was self-reported by checking as many of the following as applied: Sleep Onset Insomnia = "I have difficulty falling asleep at bedtime," Sleep Maintenance Insomnia = "After falling asleep, I wake up during the night and have difficulty getting back to sleep," Terminal Insomnia = "I wake up too early in the morning and cannot get back to sleep," Nonrestorative Sleep = "I do not feel refreshed when I get up in the morning."

Self-definition was used for the following reasons: (1) There is, at present, no uniformly accepted operational definition for the presence of insomnia (e.g., Fichten et al., 2000; Lichstein et al., 2003); (2) Individuals with similarly problematic sleep parameters self-define as either having or not having insomnia (Fichten et al., 1995); (3) A recent policy statement by the Standards of Practice Committee of the American Sleep Disorders Association (1995) suggested little role for physiological measures such as PSG in the assessment of insomnia. People complain about sleep and it is, in fact, this complaint that is of primary interest to clinicians and policy makers.

\section{Measures}

\section{Demographic}

Background Information Form. This brief questionnaire provides socio-economic, personal, and demographic descriptors (e.g., age, sex, education).

\section{Sleep}

Sleep Questionnaire. This consisted of a modified and abbreviated version of the retrospective questionnaire used in previous investigations (Fichten et al., 1995, 1998). It inquires about typical sleep experiences, including sleep parameters such as sleep onset latency, frequency of nocturnal arousals, total wake time, sleep needed, total sleep time, sleep medication taken, and aspects of sleep lifestyle such as bedtime, time when fell asleep, time of wake up, and time when out of bed. The information provided allows us to (1) compute sleep efficiency scores (\% of bedtime spent asleep) and (2) to obtain ratings of respondents' subjective perceptions of the their sleep quality and of their daytime functioning on 10-point Likert-type scales. 
Scores based on this measure have acceptable psychometric properties for research use. Test-retest correlations indicate reasonable temporal stability ( $r$ values for variables used in this investigation range from 0.58 to 0.84 ), and the pattern of correlations among variables shows logical, highly significant relationships (Fichten et al., 1995). Our convergent validity data indicate significant and high correlations between corresponding scores on the Sleep Questionnaire and on 7 days of self-monitoring on a daily sleep diary (e.g., total sleep time, $r(156)=0.82, p<0.001$; total wake time, $r(146)=0.72, p<0.001$; sleep efficiency, $r(154)=0.77, p<.001)($ Libman et al., 2000).

Structured Sleep and Medical History. A modified version of the clinical instrument developed by Lacks (1987) provides information on inclusion and exclusion criteria, parasomnias, physical disorders, sleep phase disorder, medication use, as well as use of hypnotics and sedatives. Most questions require a Yes/No answer with prompts in cases of suspected difficulty. This measure has been successfully used in studies of sleep and aging (Fichten et al., 1995; Libman et al., 1997a,b).

Daily Sleep Diary. This is a 15-item revision of Lacks' (1987) measure, which allows participants to monitor their sleep experience on a daily basis. Scores based on this measure have acceptable psychometric properties for research use (cf. Fichten et al., 1995, 1998; Libman et al., 1997b, 2000).

\section{Daytime Sleepiness/Fatigue}

Stanford Sleepiness Scale (SSS). This scale, developed by Hoddes et al. (1973), is frequently used to assess subjective perceptions of daytime sleepiness and fatigue. It consists of a 7-point Guttman scaled item ranging from 1 (feeling active and vital; alert; wide awake) to 7 (lost struggle to remain awake). Respondents select the one option which best describes how sleepy they feel.

Epworth Sleepiness Scale (ESS). This well-known brief self-administe red questionnaire of the behavioral aspects of sleepiness was constructed by Johns (1991) to evaluate self-reports of behavioral sleep tendency. Higher scores indicate greater sleepiness.

Chalder Fatigue Scale. (Chalder et al., 1993). This is an 11-item selfrating scale developed to measure severity of experienced fatigue. The original version provided four response options: 1 "not at all," 2 "no more than usual," 3 "more than usual," and 4 "much more than usual." This was revised for clinical use by our laboratory to use a 6-point Likert scale where $1=$ strongly disagree and $6=$ strongly agree. The measure has two subscales to evaluate two kinds of fatigue: physical and mental. A total fatigue score is obtained by summing all items. Subscale scores can be obtained by summing 
scores on the physical fatigue and on the mental fatigue items. The test has been shown by its authors to have good reliability $(r=0.86$ for physical fatigue, and $r=0.85$ for mental fatigue) and has high internal consistency as measured by Cronbach's alpha (0.89). Validation coefficients for the fatigue scale, using the Revised Clinical Interview Schedule as applied to individuals with CFS were sensitivity 75.5 and specificity 74.5. Higher scores indicate greater fatigue.

Fatigue Severity Scale. Developed by Krupp et al. (1989), this 9-item scale assesses "disabling fatigue." The scale's authors report psychometric information which shows that the measure is internally consistent. The single score correlates well with analogue measures and it differentiated controls $($ mean $=2.3, \mathrm{SD}=0.7)$ from lupus $($ mean $=4.7, \mathrm{SD}=1.5)$ and multiple sclerosis patients (mean $=4.8, \mathrm{SD}=1.3$ ). It could also predict clinically anticipated changes in fatigue over time. The measure was also shown to be largely independent of depressive symptoms. In addition, it has also been successfully used in insomnia research (Lichstein et al., 1994).

\section{Health and Quality of Life}

SF-36 Health Survey. (Ware et al., 2000). A 36-item short-form (SF-36) was constructed to survey health status in the Medical Outcomes Study. The SF-36 was designed for use in clinical practice and research and assesses eight health domains: (1) limitations in physical activities because of health problems; (2) limitations in social activities because of physical or emotional problems; (3) limitations in usual role activities because of physical health problems; (4) bodily pain; (5) general mental health (psychological distress and well-being); (6) limitations in usual role activities because of emotional problems; (7) vitality (energy and fatigue); and (8) general health perceptions. The survey was constructed either for self-administration or for administration by a trained interviewer. Ware et al. (2000) report reliability data from various studies carried out on both patient and nonpatient samples. Reliability of the subscales ranged from 0.64 to 0.96 in different reference groups, the lowest being for psychiatric patients on the general health subscale. The SF-36 has demonstrable validity in that the subscales were found to correlate with ability to work, utilization of health services, as well as other mental health and quality of life measures. Low scores on all subscales indicate disability due to illness, while high scores indicate better functioning due to relatively good health.

\section{Psychological Adjustment}

Beck Depression Inventory (BDI-II). The 21-item BDI (Beck et al., 1996) is one of the most frequently used measures of depression. As in 
the original version, items are scored on a 4-point scale (0-3); scores are summed and produce a range from 0 to 63 . Higher scores indicate greater depression. Although three are no norms for the scale, a score over 17 is usually considered indicative of clinical depression, while scores of 16 or less are generally considered non-depressed (Burns, 1980). The scale has excellent psychometric properties (internal consistency: $r=0.92$; test-retest reliability: $r=0.93$ ).

Spielberger State-Trait Anxiety Inventory - Form Y2. (STAI; Spielberger et al., 1983). This frequently used measure consists of two separate 20 -item self-report scales for measuring trait and state anxiety. In the present investigation, only trait anxiety was evaluated. The trait measure asks people to describe how they generally feel on 4-point Likert-type scales $(1=$ almost never, $4=$ almost always). Scores range from 20 to 80 . The authors report the following means and standard deviations for the normative sample of middle-aged adults: males $M=35.06, \mathrm{SD}=8.88$, females $M=35.03, \mathrm{SD}=$ 9.31. Higher scores indicate greater anxiety.

Eysenck Personality Questionnaire - Revised - Short. (EPQ-R; Eysenck and Eysenck, 1991). This is a 48-item revision of Eysenck and Eysenck's (1968) well-known Eysenck Personality Inventory (EPI). This reliable and valid empirically based questionnaire is among the most frequently used measures of personality (Digman, 1990). Of interest to the present investigation is the Neuroticism subscale. The authors report the following means and standard deviations for the Neuroticism subscale in the normative sample of middle-aged adults: men $M=5.50, \mathrm{SD}=3.46$, women $M=5.28$, $\mathrm{SD}=3.37$. Higher scores indicate greater Neuroticism.

Brief Symptom Inventory. (BSI; Derogatis et al., 1976). A 53-item selfreport psychological symptom inventory, the BSI has subscales for nine symptom dimensions (e.g., Depression, Anxiety, Somatization) and three global indices. It is a brief version of the SCL-90 (Derogatis, 1977) - a frequently used instrument with acceptable reliability and validity. Lower scores indicate better adjustment. Validation data indicate correlations from 0.92 to 0.98 between the symptom dimensions and global indices of the BSI and the SCL-90 (Derogatis, 1977). Lower scores indicate better adjustment.

\section{Procedure}

The research protocol was approved by the research ethics committees of both the SMBD-Jewish General Hospital and the Mount Sinai Hospital of Montreal. All participants gave informed consent.

Participants were first screened for eligibility on the telephone. Potential participants completed the Structured Sleep and Medical History interview and the following measures: Sleep Questionnaire, SF-36 Health Survey, Brief 
Symptom Inventory, Eysenck Personality Questionnaire, Spielberger StateTrait Anxiety Inventory, and Beck Depression Inventory. They also completed retrospective versions (how were you feeling on most days during the past month) of the following four questionnaires: Chalder Fatigue Scale, Fatigue Severity Scale, Epworth Sleepiness Scale, and Stanford Sleepiness Scale.

At the end of the interview and questionnaire session all participants were given seven daily rating scale packages to take home to complete first thing in the morning for seven consecutive days. Measures that participants completed on these seven consecutive days were the Daily Sleep Diary (based on previous night) and diary versions (based on the previous day) of the following four measures: Epworth Sleepiness Scale, Fatigue Severity Scale, Stanford Sleepiness Scale, and Chalder Fatigue Questionnaire.

Participants were also sent to the sleep laboratory of the Mt. Sinai Hospital (Montreal) for one overnight session. This took place anywhere from 1 week to 6 months after the interview/evaluation session and was dependent on sleep lab and participant availability. Sleep was monitored overnight by polysomnography (PSG). During the following day, while still at Mt. Sinai, participants were asked to complete the self-report measures of the daily rating scale package at 8:00 AM, 10:00 AM, 12:00 noon, and 2:00 PM.

Once results of the PSG testing were known, participants met with the team respirologist. In addition, a senior research team member gave participants detailed feedback about the results of the study. If any sleep disturbances were detected, appropriate referrals were made for either treatment or further assessment.

Given the lengthy and demanding nature of this study, a large number of participants withdrew before completing all tasks. Although all 81 participants completed the initial interview and questionnaire session, only 72 completed the 7 days of daily monitoring, and only 55 attended the sleep laboratory session. The attrition rate was greatest for the control group, where only $63 \%$ of participants completed the sleep laboratory phase of testing. For both clinical groups the corresponding value was $70 \%$.

\section{RESULTS}

\section{Data Analytic Strategies}

To compare CFS, Narcolepsy, and Control samples a series of multivariate analysis of variance comparisons (MANOVAs) were conducted. When significant differences were indicated by the MANOVA, a series of univariate analyses of variance comparisons (ANOVA) were performed to 
further clarify the results. Whenever indicated, post hoc tests were performed (Tukey HSD test). Some measures of sleep, sleepiness, and fatigue were administered up to 12 times: initial interview (1); daily monitoring (7); sleep laboratory (4). To ensure the most comprehensive data set, scores for all occasions where data were available were averaged to yield a single score for each participant. Sample sizes vary slightly for the different analyses because of missing data.

In addition to the MANOVA, multivariate analysis of covariance (MANCOVA) comparisons were performed to investigate the contribution made by the psychological adjustment variables of anxiety and depression to the dependent variables. To determine strength of effect, variance that the variable "group membership" (i.e., Narcolepsy, CFS, or Control) accounted for in a variety of dependent variables is presented as the Eta squared statistic $\left(\eta^{2}\right)$ obtained following MANCOVAs. When the MANCOVAs were significant, ANCOVA comparisons using depression and anxiety scores as covariates were also carried out to ensure that the differences found between the samples were not due uniquely to psychological adjustment.

\section{Prevalence of Sleep Disorder}

Following the PSG assessment, a large number of participants fulfilled the diagnostic criteria for a medically based sleep disorder (cf. Diagnostic Classification Steering Committee, 1990). The proportion of participants in each group receiving a diagnosis for Sleep Apnea, Sleep Hypopnea, PLMD, and RLS is presented in Table I. In the case of the Narcolepsy group, $43 \%$ fulfilled criteria for at least one medically based sleep disorder. Fifty-eight percent of the CFS group fulfilled criteria for a sleep disorder, as did $13 \%$ of the Control group. Small cell sizes did not allow for nonparametric tests to be carried out on individual variables. Therefore, to test the hypothesis that there would be a higher prevalence of medically based sleep disorders in the CFS sample than in the Control group, the total prevalence of diagnosed medically based sleep disorders was compared. The chi-square test with Yates' correction for continuity was significant, $\chi \chi^{2}(1)=5.99, p<0.05$, indicating that the CFS group had a significantly higher prevalence of sleep disorder than the Control group. Similar evaluations showed that scores in the Narcolepsy sample were not significantly different from either the CFS or Control groups.

\section{Insomnia, Sleep, and Daytime Functioning}

To evaluate similarities and differences among the three groups on Sleep Questionnaire insomnia, sleep and daytime functioning measures MANOVA 
Table I. Prevalence of Diagnosed Sleep Disorder Following PSG: Frequencies

\begin{tabular}{lccc}
\hline \multicolumn{1}{c}{ Disorder } & \multicolumn{3}{c}{ Group } \\
\cline { 2 - 4 } & Narcolepsy & CFS & Control \\
\hline Breathing disorders & & & \\
$\quad$ Sleep apnea & $3 / 14$ & $4 / 26$ & $0 / 15$ \\
$\quad$ Sleep hypopnea & $2 / 14$ & $7 / 26$ & $2 / 15$ \\
Movement disorders & $1 / 14$ & $3 / 26$ & $0 / 15$ \\
$\quad$ Periodic leg movement disorder & $0 / 14$ & $1 / 26$ & $0 / 15$ \\
$\quad$ Restless legs syndrome & $6 / 14$ & $15 / 26$ & $2 / 15$ \\
Total prevalence of any diagnosed sleep & & & \\
$\quad$ disorder following PSG & & & \\
\hline
\end{tabular}

comparisons were carried out separately on nighttime and daytime variables. Both were significant, $F(6,148)=12.547, p<0.001$ and $F(10,146)=$ $9.390, p<0.001$, respectively. Table II presents the means standard deviations for each group on the three nighttime variables and the five daytime variables as well as univariate ANOVA and ANCOVA test results and Tukey HSD post hoc tests.

Results on nighttime variables in Table II show that all three ANOVA and ANCOVA comparisons were highly significant (Have Insomnia, Sleep Quality, Sleep Satisfaction). Tukey HSD test results in Table II show that both the CFS and Narcolepsy groups had worse scores than the Control group, and that the two clinical groups did not differ significantly. Nevertheless, it is noteworthy that $86 \%$ of the CFS sample, $55 \%$ of the Narcolepsy sample, and $8 \%$ of the Control sample reported that they had insomnia. Frequency counts show that $22 \%$ of the CFS sample indicated that they had all four types of insomnia and that almost two-thirds indicated that they had at least three of the four varieties of insomnia: Sleep Onset Insomnia, Maintenance Insomnia, Terminal Insomnia, Nonrestorative Sleep (cf. American Psychiatric Association, 1994). Only 2 of the 37 individuals, $0.5 \%$, indicated that they had none of the four types of insomnia problems. Breakdowns in Table II show that the CFS group's percentages were the highest for all four subtypes of insomnia. The rate for Nonrestorative Sleep (89\%), a type of insomnia that may not have been considered insomnia by respondents, is especially remarkable

Similarly, the ANOVAs, ANCOVAs, and Tukey HSD tests show significantly worse ratings on all the daytime variables for both the CFS and Narcolepsy samples when compared to the Control sample. The CFS group's ratings were significantly worse than the Narcolepsy sample's on only 1 of the 5 daytime variables: Tired During the Day rating.

A separate MANOVA on the six Daytime Fatigue and Sleepiness variables in Table II also showed a significant group main effect, $F(12,142)=$ 


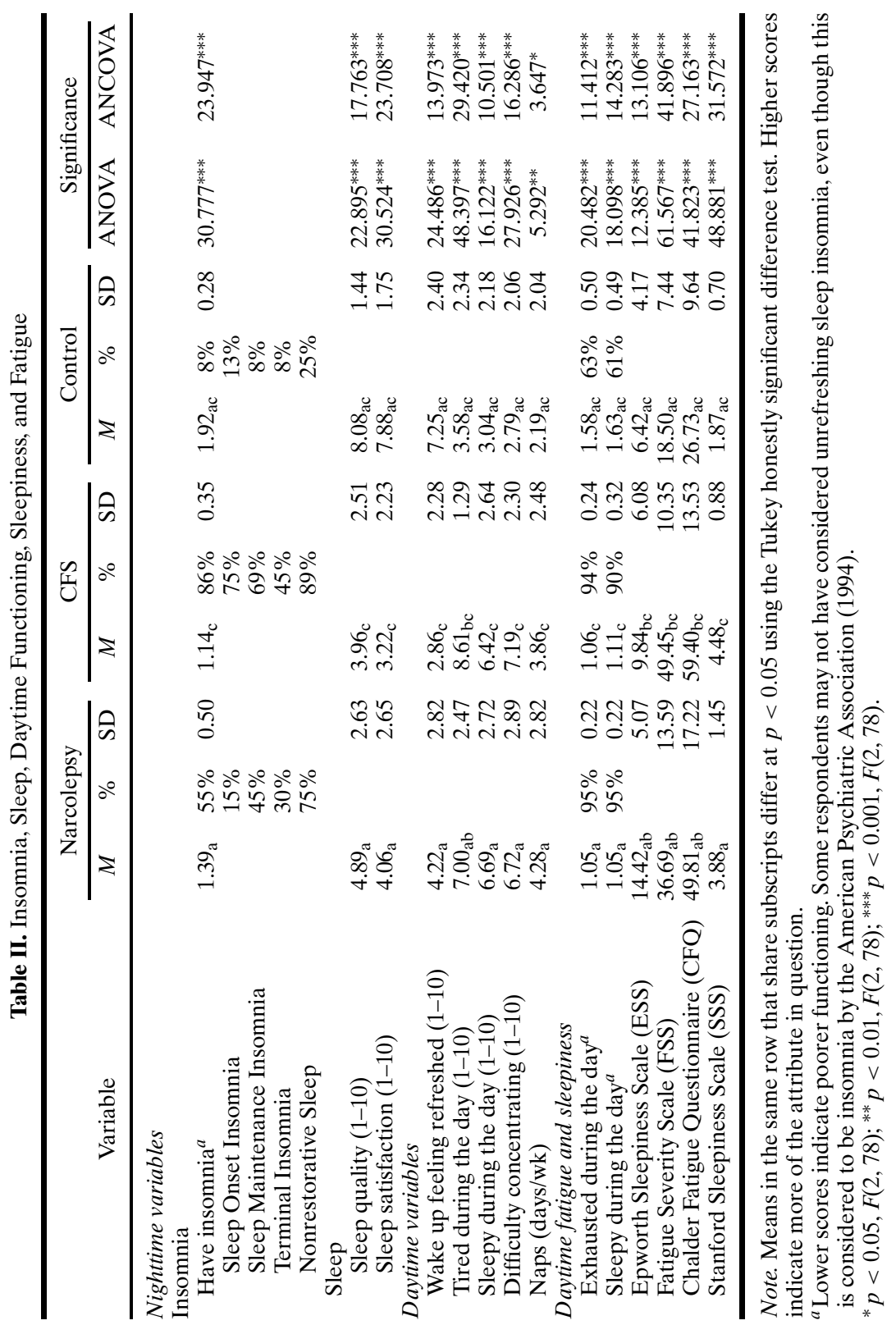


12.577, $p<0.001$. Two of the variables in Table II are from the Structured Sleep and Medical History ("Exhausted During the Day" and "Sleepy During the Day") and the remaining four represent repeated administrations of the four ongoing fatigue and sleepiness measures.

As was the case for the previous analyses, it can be seen in Table II that all ANOVA, ANCOVA, and Tukey HSD tests for all six variables were significant. These show significantly worse ratings of daytime functioning for both CFS and Narcolepsy participants compared to Controls, indicating that the Narcolepsy and CFS samples were both significantly more tired and sleepy than their Control counterparts. The two clinical groups differed from each other in three instances: the CFS group's ratings were significantly worse than the Narcolepsy sample's on the Fatigue Severity Scale and the Chalder Fatigue Questionnaire while the reverse was true for the Epworth Sleepiness Scale.

\section{Sleep Parameters and Practices}

Results on participants' sleep parameters and practices (based on the Daily Sleep Diaries) in Table III show that both the MANOVA, $F(22,124)=$ $3.364, p<0.001$, and the MANCOVA, $F(22,112)=2.425, p<0.001$, on the 11 variables were significant. The ANOVAs and the Tukey HSD test demonstrate significant differences between the CFS and Control samples on 6 of the 11 variables, indicating that participants in the CFS group took longer to fall asleep, woke up more frequently during the night, spent more time in bed awake, felt that they need more sleep, went to bed earlier, and had lower sleep efficiency scores than those in the Control group. The Narcolepsy group differed significantly from the Control group on only 2 of the 6 variables: Frequency of Nocturnal Arousals and Total Wake Time. The CFS group was significantly different from the Narcolepsy sample on only 1 variable: they took significantly longer to fall asleep than those in the Narcolepsy group.

The ANCOVA was significant only on five of these six variables. With the exception of the variable "Sleep Needed," when the effects of depression and anxiety on the sleep parameters and practices variables were statistically controlled for, the overall pattern of results did not change. This indicates that apart from the perception of how much sleep one needs, the differences between the samples on these variables were not due uniquely to psychological adjustment. In fact, group membership accounted for a larger proportion of the variance on the sleep parameters and practices variables $\left(\eta^{2}=0.323\right)$ than did either anxiety $\left(\eta^{2}=0.137\right)$ or depression $\left(\eta^{2}=0.170\right)$. 
Table III. Sleep Parameters and Practices: Daily Sleep Diary

\begin{tabular}{|c|c|c|c|c|c|c|c|c|}
\hline \multirow[b]{2}{*}{ Variable } & \multicolumn{2}{|c|}{ Narcolepsy } & \multicolumn{2}{|c|}{ CFS } & \multicolumn{2}{|c|}{ Control } & \multicolumn{2}{|c|}{ Significance } \\
\hline & $M$ & SD & $M$ & SD & $M$ & SD & ANOVA & ANCOVA \\
\hline $\begin{array}{l}\text { Sleep onset } \\
\text { latency (hrs) }\end{array}$ & $0.43_{b}$ & 0.55 & $1.31_{\mathrm{bc}}$ & 0.99 & $0.45_{c}$ & 0.58 & $11.319^{* * *}$ & $7.756^{* * *}$ \\
\hline $\begin{array}{c}\text { Frequency of } \\
\text { nocturnal } \\
\text { arousals }\end{array}$ & $2.50_{\mathrm{a}}$ & 1.90 & $2.17_{\mathrm{c}}$ & 1.27 & $0.99_{\mathrm{ac}}$ & 0.85 & $7.754^{* * *}$ & $8.310^{* * *}$ \\
\hline $\begin{array}{l}\text { Total wake time } \\
\text { (hrs) }\end{array}$ & $0.91_{\mathrm{a}}$ & 0.97 & $0.81_{c}$ & 0.50 & $0.25_{\mathrm{ac}}$ & 0.21 & $8.283^{* * *}$ & $5.104^{* *}$ \\
\hline $\begin{array}{l}\text { Sleep needed } \\
\text { (hrs) }\end{array}$ & 8.67 & 1.08 & $9.42_{c}$ & 1.55 & $8.20_{c}$ & 0.93 & $6.530^{* *}$ & 2.368 \\
\hline $\begin{array}{l}\text { Total sleep time } \\
\text { (hrs) }\end{array}$ & 7.48 & 1.56 & 7.22 & 1.63 & 7.68 & 1.03 & 0.684 & 1.252 \\
\hline $\begin{array}{l}\text { Time when went } \\
\text { to bed }\end{array}$ & 11:13 PM & $51 \mathrm{~min}$ & $10: 30 \mathrm{PM}_{\mathrm{c}}$ & $63 \mathrm{~min}$ & $11: 09 \mathrm{PM}_{\mathrm{c}}$ & $53 \mathrm{~min}$ & $4.290^{*}$ & $3.319^{*}$ \\
\hline $\begin{array}{l}\text { Time when fell } \\
\text { asleep }\end{array}$ & $11: 36 \mathrm{PM}$ & $67 \mathrm{~min}$ & $11: 59 \mathrm{PM}$ & $81 \mathrm{~min}$ & 11:36 PM & $60 \mathrm{~min}$ & 0.318 & 0.303 \\
\hline Time wake up & 7:10 AM & $89 \mathrm{~min}$ & 7:05 AM & $83 \mathrm{~min}$ & 6:48 AM & $62 \mathrm{~min}$ & 0.152 & 0.589 \\
\hline $\begin{array}{l}\text { Time get out of } \\
\text { bed }\end{array}$ & 7:14 AM & $99 \mathrm{~min}$ & 7:51 AM & $90 \mathrm{~min}$ & 7:02 AM & $61 \mathrm{~min}$ & 0.785 & 0.243 \\
\hline $\begin{array}{l}\text { Sleep medication } \\
\text { taken } \\
\text { (days/week) }\end{array}$ & 1.83 & 0.50 & 1.72 & 0.23 & 1.59 & 0.35 & 2.457 & 2.175 \\
\hline $\begin{array}{l}\text { Sleep efficiency } \\
\text { (time } \\
\text { asleep/time in } \\
\text { bed) }\end{array}$ & 0.79 & 0.19 & $0.69_{\mathrm{c}}$ & 0.15 & $0.88_{c}$ & 0.00 & $12.516^{* * *}$ & $8.586^{* * *}$ \\
\hline
\end{tabular}

Note. Means in the same row that share subscripts differ at $p<0.05$ using the Tukey honestly significant difference test.

${ }^{*} p<0.05, F(2,72) ;{ }^{* *} p<0.01, F(2,72) ;{ }^{* * *} p<0.001, F(2,72)$.

\section{Perceived Health Functioning}

In Table IV results are presented for participants' perceived health functioning as measured by the SF-36 Health Survey's eight subscales. Both the MANOVA, $F(16,136)=7.147, p<0.001$, and the MANCOVA, $F(16,126)=5.308, p<0.001$, were significant. On both the ANOVAs and ANCOVAs, six of the eight comparisons were significant (all except "Role Emotional" and "Mental Health"). The Tukey HSD test shows that the CFS sample's scores were significantly worse than those of the Control group on all six. The Narcolepsy sample's scores were worse than those of the Control sample on five of these six variables. The CFS sample's scores, however, were significantly worse than those of the Narcolepsy sample on all six variables. Group membership accounted for a larger proportion of the variance on the SF-36 health functioning variables $\left(\eta^{2}=0.403\right)$ than did either anxiety $\left(\eta^{2}=0.192\right)$ or depression $\left(\eta^{2}=0.198\right)$. 
Table IV. Perceived Health Functioning: Mean SF-36 Health Survey Scores

\begin{tabular}{|c|c|c|c|c|c|c|c|c|}
\hline \multirow[b]{2}{*}{ Subscale } & \multicolumn{2}{|c|}{ Narcolepsy } & \multicolumn{2}{|c|}{ CFS } & \multicolumn{2}{|c|}{ Control } & \multicolumn{2}{|c|}{ Significance } \\
\hline & $M$ & SD & $M$ & SD & $M$ & SD & ANOVA & ANCOVA \\
\hline $\begin{array}{l}\text { Physical } \\
\text { functioning }\end{array}$ & $71.25_{\mathrm{ab}}$ & 28.88 & $44.26_{b c}$ & 22.26 & $89.38_{\mathrm{ac}}$ & 20.34 & $26.661^{* * *}$ & $17.594^{* * *}$ \\
\hline Role physical & $38.75_{\mathrm{ab}}$ & 42.52 & $7.35_{\mathrm{bc}}$ & 17.97 & $83.33_{\mathrm{ac}}$ & 31.85 & $44.569^{* * *}$ & $25.892^{* * *}$ \\
\hline Bodily pain & $68.05_{\mathrm{b}}$ & 29.26 & $40.47_{\mathrm{bc}}$ & 24.09 & $84.33_{\mathrm{c}}$ & 20.24 & $23.744^{* * *}$ & $14.627^{* * *}$ \\
\hline General health & $56.25 \mathrm{ab}$ & 30.20 & $28.74_{b c}$ & 16.36 & $75.50_{\mathrm{ac}}$ & 19.38 & $34.227^{* * *}$ & $23.212^{* * *}$ \\
\hline Vitality & $38.75_{\mathrm{ab}}$ & 20.19 & $20.07_{b c}$ & 17.08 & $63.54 \mathrm{ac}$ & 26.88 & $29.328^{* * * *}$ & $16.759^{* * *}$ \\
\hline $\begin{array}{l}\text { Social } \\
\quad \text { functioning }\end{array}$ & $58.75_{\mathrm{ab}}$ & 30.10 & $30.15_{b c}$ & 23.86 & $88.54_{a c}$ & 18.40 & $41.332^{* * *}$ & $24.475^{* * *}$ \\
\hline Role emotional & 48.33 & 45.21 & 58.82 & 44.24 & 76.39 & 37.40 & 2.498 & 1.025 \\
\hline Mental health & 65.15 & 16.98 & 62.00 & 17.98 & 73.67 & 22.28 & 2.662 & 0.069 \\
\hline
\end{tabular}

Note. Higher scores indicate better functioning due to good health. Means in the same row that share subscripts differ at $p<0.05$ using the Tukey honestly significant difference test.

${ }^{* * *} p<0.001, F(2,78)$.

Comparisons of subscale scores for each group with available normative data indicates that Control sample scores fall within the normative range of the SF-36 (i.e., within one standard deviation) on all subscales. Narcolepsy sample scores fall one standard deviation below the normative mean on three of the eight subscales ("Role Physical," "Vitality," and "Social Functioning"), indicating poorer health functioning with respect to physical and social aspects. Scores of the CFS sample are outside the normative range on six subscales (all except Role Emotional and Mental Health). Most notably, the CFS sample scores two standard deviations below the normative mean on three subscales: "Role Physical," "General Health," and "Social Functioning." These indicate substantially poorer health functioning with respect to physical and social aspects.

\section{Psychological Adjustment}

Table $\mathrm{V}$ presents means and test results on the five measures of psychological adjustment. The MANOVA was significant, $F(10,132)=4.049$, $p<0.001$, as well as all five ANOVAs. Tukey HSD test results show that the Narcolepsy and CFS groups did not differ significantly on any of the psychological adjustment variables, although the CFS sample scored significantly worse than the Control group on all five variables while the Narcolepsy group scored worse on two of these.

When scores for each group were compared to available normative data for the measures, scores of the Control group consistently fell within one standard deviation of the normative group mean. The Narcolepsy and 


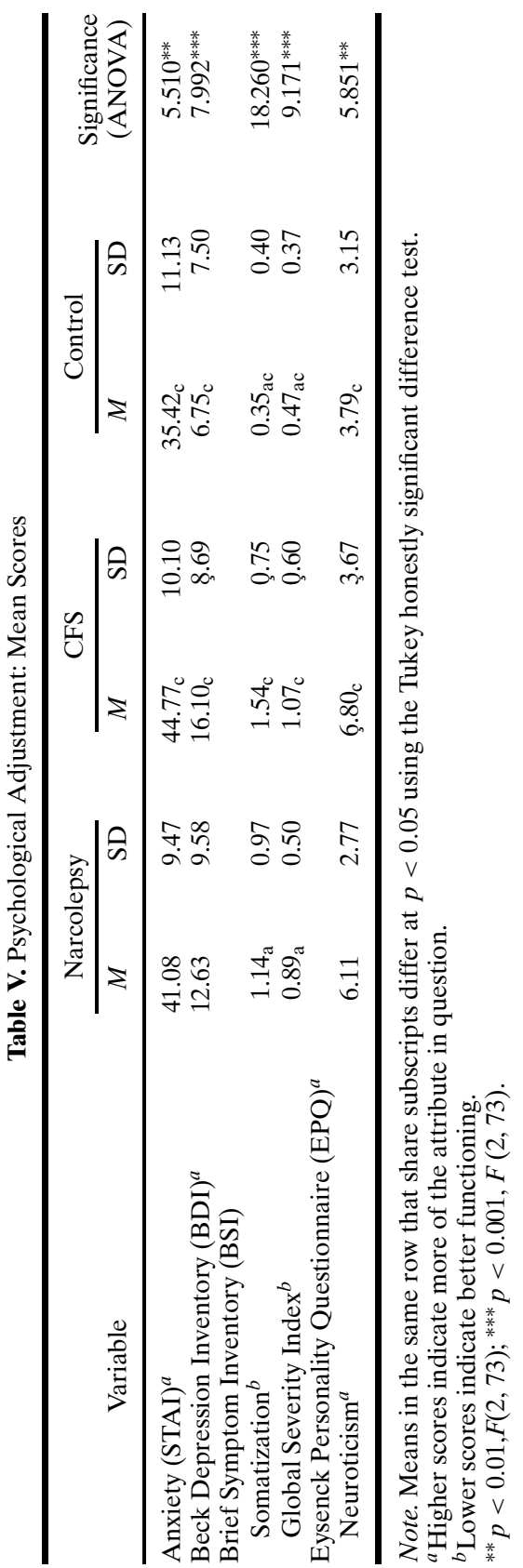


CFS samples, however, generally scored one standard deviation above the normative mean for the Spielberger State-Trait Anxiety Inventory, indicating higher anxiety for both these groups. In addition, both the Narcolepsy and CFS groups scored two standard deviations above the normative mean on both Brief Symptom Inventory measures, indicating higher Somatization and poorer Overall Psychological Adjustment for both groups. Finally, it is worth noting that none of the groups scored outside the normative range on measures of depression or neuroticism.

\section{DISCUSSION}

\section{Nature and Prevalence of Sleep Disorders in CFS}

The CFS sample studied here had, as predicted, a very high incidence $(58 \%)$ of primary sleep disorder such as sleep apnea/hypopnea syndrome and restless legs syndrome/periodic limb movement disorder. Although we expected relatively high rates, the prevalence of these in CFS was surprising. Of the CFS sample, 15\% had sleep apnea, 27\% had sleep hypopnea, 12\% had periodic limb movement disorder, and $4 \%$ has restless legs syndrome. In fact, it was less than half of the sample who did not receive a diagnosis for some kind of significant physiological sleep disorder.

The incidence of sleep disorder in the CFS sample was substantially greater than that in the control sample. Furthermore, on many sleep-related aspects individuals with CFS were not different from individuals with the physiologically based sleep disorder of narcolepsy. In fact, in some ways it was the CFS sample that had a higher incidence of sleep disruption than the narcolepsy sample. Considering the magnitude of the medically based sleep-related problems, it is notable that prior to participating in this study, neither the CFS patients nor their physicians had been aware that they had these disorders.

The CFS sample also had very high rates of self-reported insomnia (86\%). In addition, $89 \%$ of the CFS sample also indicated that they woke up feeling unrefreshed. The high rate of insomnia (i.e., disorder in initiating and maintaining sleep, including nonrestorative sleep-cf. American Psychiatric Association, 1994) is consistent with findings in the literature and attest to the debilitating nature of the sleep disruption experienced in this population (Hardt et al., 2001; Yehuda and Mostofsky, 1997). Indeed, only $0.5 \%$ of the CFS sample indicated that they had none of the four types of insomnia problems.

The CFS sample, on the whole, had a wide variety of sleep-related complaints. Besides waking up feeling unrefreshed and having significantly 
prolonged sleep onset latencies, the CFS sample woke up frequently during the night, and spent a large amount of time in bed at night not sleeping. They also rated their sleep quality as poor and were less satisfied with their sleep than those in the other two groups. This, despite the fact that the CFS sample went to bed and got out of bed at roughly the same time as both their narcolepsy and control group counterparts. In essence, the CFS sample displayed the characteristic signs of poor overall sleep efficiency seen in persons with insomnia.

Thus, our findings indicate that more than half of the CFS population may have a diagnosable physiologically based sleep disorder, and that virtually all individuals with CFS may have insomnia. Currently, research is ongoing in our laboratory to investigate the impact of treating the sleep disorder on chronic fatigue as well as daytime functioning. Very preliminary findings indicate that general functioning and perceived quality of life improve with treatment, but that the fundamental fatigue aspect is not resolved (Creti et al., 2003). Although the final evidence is not yet in, contrary to the conclusion drawn by Le Bon et al. (2000), who also showed high rates of disordered sleep, we believe that the present findings and our ongoing research suggest that primary sleep disorder and insomnia are comorbidities in CFS and important targets in treatment.

\section{Daytime Functioning}

It was not surprising to find that individuals with CFS were more fatigued during the day than the other two groups on most measures used in this study. This, after all, is the hallmark symptom in the diagnosis of CFS. It was reassuring that the CFS sample was more fatigued that those with narcolepsy, indicating that their daytime functioning, in this case, was not only worse than their healthy counterparts' but also worse than that of individuals with a known diagnosed medical disorder. Their scores on daytime sleepiness were also significantly elevated compared to the control group, although for the most part the scores of the narcolepsy sample indicated somewhat greater sleepiness. Overall, the results show that daytime functioning in the CFS sample is seriously compromised.

\section{Health and Quality of Life}

The measure designed to assess overall health functioning (SF-36 Health Survey) also evaluates quality of life. Here again, scores of the CFS sample were not only significantly poorer than those of the Control group, but also 
were below the normative range on most subscales. It is of interest to note that although individuals with narcolepsy, a clearly identifiable medical disorder, reported poor health functioning (as indicated by low scores on SF-36 subscales), participants with CFS reported even poorer health functioning on most subscales. An important difference between these two clinical groups is that for narcoleptics, their principal complaint, sleepiness, is treatable with medication that presumably mitigates the impact of their condition on their general functioning and perceived quality of life. Thus, it appears that symptoms of CFS significantly undermine many facets of these individuals' lives and impair physical and social functioning as well as participation in daily activities.

\section{Psychological Adjustment}

Overall, psychological adjustment in the CFS sample was significantly poorer than that in the control sample. There were no significant differences, however, between the two clinical groups, CFS and narcolepsy, on any of the psychological adjustment variables evaluated. Both when compared to the control sample as well as when compared to the normative data, the CFS sample scored in ways that are consistent with slightly elevated anxiety and somatization as well as generally poorer psychological adjustment. Scores of the CFS sample were also different from those of healthy controls on depression and neuroticism. It is worthy of note, however, that the scores for depression and neuroticism were well within the normative range for their age group and, in this sense, not clinically significant.

What about the popular stereotype of psychological maladjustment in CFS symptom sufferers? It was, indeed, the case that individuals with CFS were significantly more anxious and presented more somatic complaints than their healthy control counterparts. However, despite their experience of debilitating fatigue and generally poor quality of life, people with CFS, did not score within the clinically maladjusted range on measures of depression or neuroticism; this latter is believed to be a biologically based predisposition (cf. Eysenck, 1952). It is notable that they did not differ significantly from individuals with narcolepsy, who also had significantly worse scores on some measures than did the control subjects.

Although the lack of significant differences between the CFS and narcolepsy samples is intriguing, it is possible that this reflects a lack of power (Type II error) rather than a real similarity between these groups. Nonetheless, results from other studies support our findings of psychological maladjustment in well-recognized medically based disorders. For example, in an investigation of psychological functioning in persons with narcolepsy, Mastin 
(1999) reported that in addition to experiencing excessive sleepiness, they also had significantly higher levels of depression, anxiety, and overall affective disturbance. Taken together with the presence of sleep disruption in both clinical samples, the similarities noted in the present study could reflect disruption of HPA axis function.

Additionally, in a pilot investigation of one of our team members (Bailes et al., 2001), individuals with diagnosed sleep apnea were are also found to have distinctive psychological adjustment profiles. Of course many medical patients tend to have poorer psychological adjustment scores than healthy controls (e.g., Dattore et al., 1980). Despite such findings, individuals with narcolepsy, sleep apnea, or other medical illnesses are not typically told that their problem is "all in their head" or psychosomatic, as are people with CFS (Caplan, 1998).

\section{Limitations}

An obvious limitation of the present study is the lack of power due to small sample sizes for even moderate group differences to emerge (Cohen, 1988). However, the well-documented expense related to laboratory sleep research (Komaroff and Fagioli, 1996), as well as the difficulties regularly encountered with subject attrition in such extensive, demanding, and lengthy investigations make small sample size an unfortunate, but common consequence. Nevertheless, although some differences between the samples may not have been revealed, the comprehensive descriptive data collected make an important contribution to CFS research and form the basis for future investigations. We also hope that our findings have heuristic value, particularly in the area of intervention. Furthermore, the unique comparison of a CFS sample to a narcolepsy sample, which has sleep-wake disorders and psychological aspects in common, but where the neuropathology is well described, as well as to a Control group sampled from the general population, adds a new conceptual dimension to issues of etiology, maintenance, and consequence in CFS research.

Another potential limitation is the possible presence of lifetime comorbid conditions in the CFS sample that were not controlled for. With the exception of fibromyalgia, we did exclude participants based on current comorbid conditions known to affect sleep and/or daytime functioning. But we were not able to control for the presence of other illnesses over the lifetime of CFS participants. Although it would be desirable to sample from a pure CFS population in terms of internal validity, external validity would undoubtedly be compromised as comorbid conditions are a well-known confound in the presentation and diagnosis of CFS (cf. Le Bon et al., 2000). 


\section{CONCLUSIONS}

Our findings highlight the significant amount of sleep disruption in CFS due both to medically based disorders such as sleep apnea/hypopnea syndrome, restless legs syndrome/periodic limb movement disorder (RLS/ PLMD), as well as to insomnia. Sleep disorders such as apnea/hypopnea syndrome are commonly overlooked or misdiagnosed in medical practice (e.g., Kapsimalis and Kryger, 2002; Young et al., 1996), as was the case with individuals in our CFS sample. On the basis of our findings, we recommend that physicians refer their patients with a probable diagnosis of CFS for overnight PSG evaluation.

The pattern of psychological disturbance in individuals with CFS is very similar to that in narcolepsy, a neurological disorder involving sleep. Notably, the slightly elevated depression and neuroticism found in those with CFS relative to normal controls were within the normative range. As postulated by others (e.g., Nicassio et al., 1999), the psychological disturbances seen in CFS may well be the result of living with a chronic illness that is poorly recognized or understood.

\section{ACKNOWLEDGMENTS}

This research was carried out at the both the Jewish General Hospital and the Mount Sinai Hospital in Montreal, Quebec, with the assistance of a grant from the Canadian Institutes of Health Research.

\section{REFERENCES}

American Psychiatric Association (1994). Diagnostic and Statistical Manual of Mental Disorders, 4th ed., American Psychiatric Association, Washington, DC.

Atlas Task Force of the American Sleep Disorders Association (1993). Recording and scoring leg movements. Sleep 16: 749.

Bailes, S., Baltzan, M., Alapin, I., Fichten, C. S., and Libman, E. (2003). Different but Equal: Symptom Presentation in Women and Men With Sleep Apnea, Manuscript submitted for publication.

Bailes, S., Baltzan, M., Alapin, I., Schondorf, R., Fossey, M., Fichten, C. S., and Libman, E. (2001, May). Sleep Quality and Psychological Adjustment in Chronic Fatigue, Presentation at the Canadian Sleep Society convention, Ottawa, Canada (Summary in 2001 Canadian Sleep Odyssey Conference Program, p. 46).

Beck, A., Steer, R., and Brown, G. (1996). BDI-II: Beck Depression Inventory Manual, 2nd ed., The Psychological Corporation, San Antonio, TX.

Bootzin, R. R., and Nicassio, P. M. (1978). Behavioral treatments for insomnia. In Hersen, M., Eisler, R., and Miller, P. (Eds.), Progress in Behavior Modification, Vol. 6, Academic Press, New York, pp. 1-45. 
Burns, D. D. (1980). Feeling Good: The New Mood Therapy, Avon Books, New York, pp. 22-23. Caplan, C. (1998). Chronic fatigue syndrome or just plain tired? Can. Med. Assoc. J. 159: 519.

Chalder, T., Berelowitz, G., Pawlikowska, T., Watts, L., Wessely, S., Wright, D., and Wallace, E. P. (1993). Development of a fatigue scale. J. Psychosom. Res. 37(2): 147-153.

Cleare, A. J. (2003). The neuroendocrinology of chronic fatigue syndrome. Endocrine Reviews 24: $236-252$.

Cohen, J. (1988). Statistical Power Analysis for the Behavioral Sciences, 2nd ed., Erlbaum, Hillsdale, NJ.

Creti, L., Libman, E., Bailes, S., Fichten, C. S., Jastremsky, M., Morin, N., Baltzan, M., Schondorf, R., Amsel, R., and Spector, I. (2003, November). Treatment of Sleep Disorders in Chronic Fatigue Syndrome: Cbt, Medical Treatment or Both? Presented at the Association for Advancement of Behavior Therapy (AABT), Boston.

Dattore, P. J., Shontz, F. C., and Coyne, L. (1980). Premorbid personality differentiation of cancer and noncancer groups: A test of the hypothesis of cancer proneness. J. Consult. Clin. Psychol. 48: 388-394.

David, A. S., Wessely, S., and Pelosi, A. J. (1988). Myalgic encephalomyelitis or what? Lancet 2: $100-101$.

Derogatis, L. R. (1977). The Psychopathology Rating Scale: A Brief Description. Unpublished manuscript.

Derogatis, L. R., Rickels, K., and Rock, A. F. (1976). The SCL-90 and the MMPI: A step in the validation of a new self-report scale. Br. J. Psychiatry 128: 280-289.

Diagnostic Classification Steering Committee (1990). ICSD -International Classification of Sleep Disorders: Diagnostic and Coding Manual, American Sleep Disorders Association, Rochester, MI.

Digman, J. M. (1990). Personality structure: Emergence of the 5-Factor model. Ann. Rev. Psychol. 41: 417-440.

Eysenck, H. J. (1952). The Scientific Study of Personality, Routledge \& Kenan Paul, London.

Eysenck, H. J., and Eysenck, S. B. G. (1968). Manual: Eysenck Personality Inventory, Educational and Industrial Testing Service, San Diego, CA.

Eysenck, H. J., and Eysenck, S. B. G. (1991). Manual of the Eysenck personality Scales (EPS Adult), Hodder \& Stoughton, London.

Fichten, C. S., Creti, L., Amsel, R., Brender, W., Weinstein, N., and Libman, E. (1995). Poor sleepers who do not complain of insomnia: Myths and realities about psychological and lifestyle characteristics of older good and poor sleepers. J. Behav. Med. 18(2): 189223.

Fichten, C. S., Libman, E., Bailes, S., and Alapin, I. (2000). Characteristics of older adults with insomnia. In Lichstein, K. L., and Morin, C. M. (Eds.), Treatment of Late Life Insomnia, Sage, New York, pp. 37-80.

Fichten, C. S., Libman, E., Creti, L., Amsel, R., Tagalakis, V., and Brender, W. (1998). Thoughts during awake times in older good and poor sleepers - The Self-Statement Test: 60+. Cogn. Ther. Res. 22(1): 1-20.

Fukuda, K., Strauss, S. E., Hickie, I., Sharpe, M. C., Dobbins, J. G., and Komaroff, A. L. (1994). The chronic fatigue syndrome: A comprehensive approach to its definition and study. Ann. Intern. Med. 121: 953-959.

Hardt, J., Buchwald, D., Wilks, D., Sharpe, M., Nix, W. A., and Egle, U. T. (2001). Healthrelated quality of life in patients with chronic fatigue syndrome. An international study. $J$. Psychosom. Res. 51(2): 431-434.

Hoddes, E., Zarcone, V., Smythe, H., Phillips, K., and Dement, W. C. (1973). Quantification of sleepiness: A new approach. Psychophysiology 10: 431-437.

Honma, K., Hashimoto, S., Nakao, M., and Honma, S. (2003). Period and phase adjustments of human circadian rhythms in the real world. J. Biol. Rhythms 18(3): 261-270.

Johns, M. W. (1991). A new method for measuring daytime sleepiness: The Epworth Sleepiness Scale. Sleep 14(6): 540-545.

Kapsimalis, F., and Kryger, M. H. (2002). Gender and obstructive sleep apnea syndrome: Part 1: Clinical features. Sleep 25(4): 412-419. 
Kirmayer, L. J., and Robbins, J. M. (1991). Three forms of somatization in primary care: Prevalence, co-occurrence, and sociodemographic characteristics. J. Nerv. Ment. Dis. 179(11): 647-655.

Kok, S. W., Roelfsema, F., Overseem, S., Lammers, G. J., Strijers, R. L., Frolich, M., Meinders, A. E., and Pijl, H. (2002). Dynamics of the pituitary-adrenal ensemble in hypocretindeficient narcoleptic humans: Blunted basal adrenocorticotropin release and evidence for normal time-keeping by the master pacemaker. J. Clin. Endocrinol. Metab. 87(11): 50855091.

Komaroff, A. L. (1990). Minor illness symptoms: The magnitude of their burden and of our ignorance. Arch. Intern. Med. 150: 1586-1687.

Komaroff, A. L., and Fagioli, L. (1996). Medical assessment of fatigue and chronic fatigue syndrome. In Demitrack, M. A., and Abbey, S. E. (Eds.), Chronic Fatigue Syndrome - An Integrative Approach to Evaluate and Treatment, Guilford, New York.

Krupp, L. B., LaRocca, N. G., Muir-Nash, J., and Sternberg, A. D. (1989). The Fatigue Severity Scale: Application to patients with multiple sclerosis and systemic lupus erythemasis. Arch. Neurol. 46: 1121-1123.

Lacks, P. (1987). Daily sleep diary. In Lacks, P. (Ed.), Behavioral Treatment for Persistent Insomnia, Pergamon, New York, p. 165.

Le Bon, O., Fischler, B., Hoffman, G., Murphy, J. R., De Meirleir, K., Cluydts, R., and Pelc, I. (2000). How significant are primary sleep disorders in the chronic fatigue syndrome? Sleep Res. Online 3(2): 43-48.

Leese, G., Chattington, P., Fraser, W., Vora, J., Edwards, R., and Williams, G. (1996). Shortterm night-shift working mimics the pituitary-adrenocortical dysfunction in chronic fatigue syndrome. J. Clin. Endocrinol. Metab. 81: 1867-1870.

Libman, E., Creti, L., Amsel, R., Brender, W., and Fichten, C. S. (1997a). What do older good and poor sleepers do during periods of nocturnal wakefulness? The Sleep Behaviors Scale: 60+. Psychol. Aging 12(1): 170-182.

Libman, E., Creti, L., Levy, R. D., Brender, W., and Fichten, C. S. (1997b). A comparison of reported and recorded sleep in older poor sleepers. J. Clin. Geropsychol. 3(3): 199-211.

Libman, E., Fichten, C. S., Bailes, S., and Amsel, R. (2000). Sleep questionnaire vs. sleep diary: Which measure is better? Int. J. Rehabil. Health 5(3): 205-209.

Lichstein, K. L., Durrence, H. H., Reidel, B. W., and Bayen, U. J. (2001). Primary versus secondary insomnia in older adults: Subjective sleep and daytime functioning. Psychol. Aging 16(2): 264-271.

Lichstein, K. L., Durrence, H. H., Taylor, D. J., Bush, A. J., and Reidel, B. W. (2003). Quantitative criteria for insomnia. Behav. Res. Ther. 41: 427-445.

Lichstein, K. L., Wilson, N. M., Noe, S. L., Aguillard, R. N., and Bellur, S. N. (1994). Daytime sleepiness in insomnia: Behavioral, biological and subjective indices. Sleep 17(8): 693702.

Mastin, D. F. (1999). The psychosocial impact of sleepiness in a large African-American family with several narcoleptic members (Doctoral dissertation, The University of Southern Mississippi, 1999). Diss. Abstr. Int., Sect. B: Sci. Eng. 59(7B): 3703.

Moldofsky, H. (1986). Sleep and musculoskeletal pain. Am. J. Med. 81: 85-89.

Moldofsky, H., and Scarisbrick, P. (1976). Induction of neurasthenic musculoskeletal pain syndrome by selective sleep stage deprivation. Psychosom. Med. 38: 35-44.

Montplaisir, J., and Godbout, R. (1989). Restless legs syndrome and periodic movements during sleep. In Kryger, M. H., Roth, T., and Dement, W. C. (Eds.), Principles and Practice of Sleep Medicine, Saunders, Philadelphia, PA, pp. 402-409.

Morin, C. M., Rodrigue, S., and Ivers, H. (2003). Role of stress, arousal, and coping skills in primary insomnia. Psychosom. Med. 65: 259-267.

Nicassio, P. M., Schuman, C., Radojevic, V., and Weissman, M. H. (1999). Helplessness as a mediator of health status in fibromyalgia. Cogn. Ther. Res. 23(2): 181-196.

Plioplys, A. V. (2003) Differential diagnosis in medical assessment. In Jason, L. A., Fennell, P. A., and Taylor, R. R. (Eds.), Handbook of Chronic Fatigue Syndrome, Wiley, NJ, pp. $26-41$. 
Schondorf, R., Benoit, J., Wein, T., and Phaneuf, D. (1999). Orthostatic intolerance in the chronic fatigue syndrome. J. Auton. Nerv. Syst. 75: 192-201.

Schondorf, R., and Freeman, R. (1999). The importance of orthostatic intolerance in the chronic fatigue syndrome. Am. J. Med. Sci. 317: 117-123.

Siegel, J. (2000, Winter). Recent developments in narcolepsy research, an explanation for patients and the general public. Narcolepsy Netw. Newsl. 13: 1-2.

Spath-Schwalbe, E., Scholler, T., Kern, W., Fehm, H. L., and Born, J. (1992). Nocturnal adrenocorticotropin and cortisol secretion depends on sleep duration and decreases in association with spontaneous awakening in the morning. J. Clin. Endocrinol. Metabol. 75: 1431-1435.

Spielberger, C. D., Gorsuch, R. L., Lushene, R., Vagg, P. R., and Jacobs, G. A. (1983). Manual for the State-Trait Anxiety Inventory (Form Y), Consulting Psychologists Press, Palo Alto, CA.

Standards of Practice Committee of the American Sleep Disorders Association (1995). Practice parameters for the use of polysomnography in the evaluation of insomnia. Sleep 18: 55-57.

Steiger, A. (2002). Sleep and the hypothalamo-pituitary-adrenal system. Sleep Med. Rev. 6(2): $125-138$.

Taheri, S., Zeitzer, J. M., and Mignot, E. (2002). The role of hypocretins (orexins) in sleep regulation and narcolepsy. Ann. Rev. Neurosci. 25: 283-313.

Taylor, M., and Samson, W. K. (2003). The other side of orexins: Endocrine and metabolic actions. Am. J. Physiol. Endocrinol. Metab. 284: E13-E17.

Van Reeth, O., Weibel, L., Spiegel, K., Leproult, R., Dugovic, C., and Maccari, S. (2000). Interactions between stress and sleep: From basic research to clinical situations. Sleep Med. Rev. 4: 201-219.

Ware, J. E., Snow, K. K., Kosinski, M., and Gandek, B. (2000). SF-36 Health Survey: Manual and Interpretation Guide, Quality Metric Incorporated, Lincoln, RI.

Wessely, S. (1995). Neurasthenia and fatigue syndromes. In Berrios, G., and Porter, R. (Eds.), A History of Clinical Psychiatry, The Athlone Press, London, pp. 509-532.

Williams, G., Pirmohamed, J., Minors, D., Waterhouse, J., Buchan, I., Arendt, J., and Edwards, R. H. T. (1996). Dissociation of body-temperature and melatonin secretion circadian rhythms in patients with chronic fatigue syndorme. Clin. Physiol. 16(4): 327-337.

Yehuda, S., and Mostofsky, D. I. (Eds.). (1997). Chronic Fatigue Syndrome, Plenum, New York.

Young, T., Hutton, R., Finn, L., Badr, S., and Palta, M. (1996). The gender bias in sleep apnea diagnosis. Are women missed because they have different symptoms? Arch. Intern. Med. 156(21): 2445-2451. 
Copyright of Journal of Behavioral Medicine is the property of Springer Science \& Business Media B.V. and its content may not be copied or emailed to multiple sites or posted to a listserv without the copyright holder's express written permission. However, users may print, download, or email articles for individual use. 